\title{
From Mudpack to Synthetic Facelifts: Stucco and its Cousins
}

Animal dung and acrylic are among the range of materials that have been used to clad a building's exterior. Using an exterior coating to improve a building's weathering ability and appearance, builders have employed mud, cement, and, most recently, polymer-based materials. Prehistoric structures are known to have been fashioned from brush, bones, grasses, dried clay, and sod. Rudimentary shelter was thereby achieved, but, in the inexorable desire for improved living standards, people sought greater protection from rain and wind and surer control of the indoor temperature. The application of mud and dung to the outer surface of a simple structure made it less permeable to the elements. Straw or other fibrous material could be mixed in to decrease drying time and provide a matrix of greater structural integrity.

The use of coatings with a uniform appearance that are applied when wet and allowed to dry to a hard finish was well-known to the ancient Romans and is still common throughout the world. The compound that is commonly called "stuc$\mathrm{co}^{\prime \prime}$ today is a cementitious mixture in which lime, clay or rock dust, aggregate (i.e., sand or small stones), and water are combined and worked while semi-liquid. The stucco is then smoothed over a substrate that possesses enough texture to prevent delamination of the finished surface: wood slats, brick, roughened concrete or stone, or a metal mesh are widely used. The mixture dries into a hard shell and provides a smooth, watertight surface which readily takes paint or other pigmentation.

Stucco is brittle, however, and cracks can lead to leaks, chipping, and delamination of the surface. Cracking can be controlled by careful attention to the proportions of ingredients in the mix and conscientious craftsmanship when applying the wet stucco, but it remains the most challenging aspect of the material's use. The basic formula has hardly changed in the past two millenia. Stucco's ingredients occur worldwide: lime, which can be obtained by burning seashells or limestone; many varieties of aggregate; and water. Stucco is also more versatile than many other materials in that it can be used in a wide variety of climates. Constant moisture or dryness or cyclical changes in humidity and temperature affect cementitious stucco less than they affect wood, metals, or the more primitive coatings mentioned earlier.

The Modernist building aesthetic that coalesced in Europe in the early 1900s and was adopted throughout the world by midcentury favored simple building forms with minimal adornment. A building's geometrical shape was the primary expressive vehicle, taking over the role traditionally played by ornamentation. Architects favored materials that allowed a building to appear as a cleanly executed form, and stucco proved an effective medium.

The destruction wrought by World War II in Europe made the rapid rebuilding of cities an imperative. Stucco was well-suited both for resurfacing damaged buildings and for constructing new ones; something lighter in weight, less vulnerable to cracking, more energy efficient and, ideally, less expensive, was sought.

After the war, Edwin Horbach, a young German chemist employed by a building materials company, began research in his home on a material that would incorporate insulation and a flexible exterior finish. Such a material would speed the erection of buildings, provide an energy efficient skin, and be much lighter in weight than traditional building components. Previous studies had demonstrated that insulation was most effective when used as a blanket closest to the exterior of a building rather than being buried in the walls. Horbach devised a composite system which incorporated a base of rigid expanded polystyrene insulation beneath a reinforcing fabric which formed a substrate for a thin finish coating. This system could be applied over many different rigid substrates either by attaching with adhesives or screws. Horbach applied for a patent in 1959, and although the patent was never obtained, his system was used throughout northern Europe in the 1950s and 1960s. During this period, Europeans experimented with a variety of exterior cladding systems known in the building industry as exterior insulation and finish systems (EIFS). Both foam and rock fiber insulation were tried, as well as finish coatings made from mineral bases and mineral bases augmented with synthetic polymers.

The use of polymer-containing matrices in these systems affords a degree of flexibil- ity and resistance to cracking surpassing that of traditional stucco. Aggregates of varying coarseness can be used to modulate the texture of the finished surface. Since the finish coating and its mesh reinforcing fabric (usually fiberglass) are laid over plastic insulation which is relatively soft, the outer layers need to be durable. The configuration widely used today incorporates a base layer that combines Portland cement and polymers in its matrix for hardness and flexibility; the top coat contains acrylic, aggregate, and pigment, foregoing the need for a coat of paint.

EIFS were not introduced in the United States until the late 1960s, where they were regarded as a new and untried entity. Building codes would not permit their use until they were tested under differing climactic conditions and in fires. Because the features that made EIFS a hit in Europe attracted no less attention in the United States, they have become ubiquitous in the States - examples can be found on countless commercial buildings, on shopping malls, and in residential developments. The material is popular for use in refacing old buildings quickly and inexpensively, with an additional insulating bonus. Professional journals in the construction industry frequently carry articles about their proper use and application. Traditional cementitious stucco is still in use, and has been joined by a hybrid incorporating polymers and used as stucco would be, without insulation directly over wood, metal mesh, concrete block, or adobe. Although straw-reinforced cow dung is not likely to be appearing soon on a strip mall near you, the matrix-and-aggregate strategy for cladding buildings remains current. The ingredients are easily obtained and quickly combined, revealing a late twentieth-century twist on folk construction.

AlLISON I. FulTZ

FOR FURTHER READING: V. Tamburrini, "The History and Development of EIFS" and G.L. Christensen, "Full-Scale Fire Test of Various Exterior Wall Systems," in Exterior Insulation and Finish Systems (EIFS), M. F. Williams and R.G. Lampo, eds. (1995, Philadelphia, PA); A. Benjamin, The American Builder's Companion, (Dover Publications, New York, 1969; reprint of 1827 edition); and $\mathrm{K}$. Frampton, Modern Architecture: A Critical History (London, 1980). 